\title{
Classification of eigenmodes in rf cavities using the group theory
}

\author{
Shogo Sakanaka \\ Institute of Materials Structure Science, High Energy Accelerator Research Organization (KEK), \\ 1-1 Oho, Tsukuba, Ibaraki 305-0801, Japan \\ (Received 11 May 2005; published 25 July 2005)
}

\begin{abstract}
For many damped-accelerating cavities for high-intensity beams, classifications of eigenmodes according to azimuthal indices are generally insufficient due to a lack of their axial symmetry. To classify the eigenmodes in such cavities having general symmetry, the application of group theory is studied. By taking basis functions from a complete set of eigenmodes, one can form a representation of the symmetry group of the cavity. The eigenmodes can then be classified according to irreducible representations of the symmetry group. This method is particularly useful for classifying the eigenmodes in complicated cavities, and for understanding the effects of perturbations on the eigenmodes.
\end{abstract}

\section{INTRODUCTION}

Radio-frequency cavities for particle accelerators are characterized by their symmetry. High symmetry of a cavity simplifies both its design and the analysis of resonant modes. Axial symmetry about the beam axis has a distinct advantage that each eigenmode has a definite angular dependence of $\cos m \phi$ or $\sin m \phi$. This ensures that no transverse kicks can be imposed upon on-axis particles by an axially symmetric mode, usually used for beam acceleration.

In many damped-accelerating cavities [1] for highintensity beams, however, the axial symmetry is broken. For example, Fig. 1 shows a picture of the $714-\mathrm{MHz}$ damped cavity [2] for the accelerator test facility (ATF) damping ring at the High Energy Accelerator Research Organization (KEK). This cavity is equipped with four waveguide ports for damping harmful higher-order modes (HOMs). Two of the ports are located at the upper and lower positions on the upstream side, while the other two are at the left and right positions on the downstream side. Each eigenmode in this cavity generally contains several harmonic components. While characterizing the HOMs in this cavity, we encountered a problem of how we can classify, or can express the symmetry properties of, the eigenmodes. The present work was motivated by this need.

In many fields of physics, group theory [3-5] is widely used for handing the symmetry of physical systems, for instance, for classifying the atomic and molecular spectra. It is expected that the eigenmodes in the cavities can be classified elegantly by applying a similar method.

For electromagnetic problems, much consideration on the symmetry has been presented, for example, an analysis of waveguide junctions by Dicke [6], an electromagnetic theory of periodic structures by Bevensee [7], and a consideration of the symmetry in field solvers by Nelson [8]. Recently, the eigenmodes in periodic dielectric structures (so-called photonic lattices) have been studied in terms of group theory [9]. However, we can hardly find a handy document about an application of group theory to cavityeigenmode problems in a convenient form for accelerator applications. This paper aims to provide a basis for such applications. First, we introduce a matrix representation of symmetry operations for a cavity. We show that the eigenmodes in the cavity can be classified according to irreducible representations of the symmetry group. This procedure

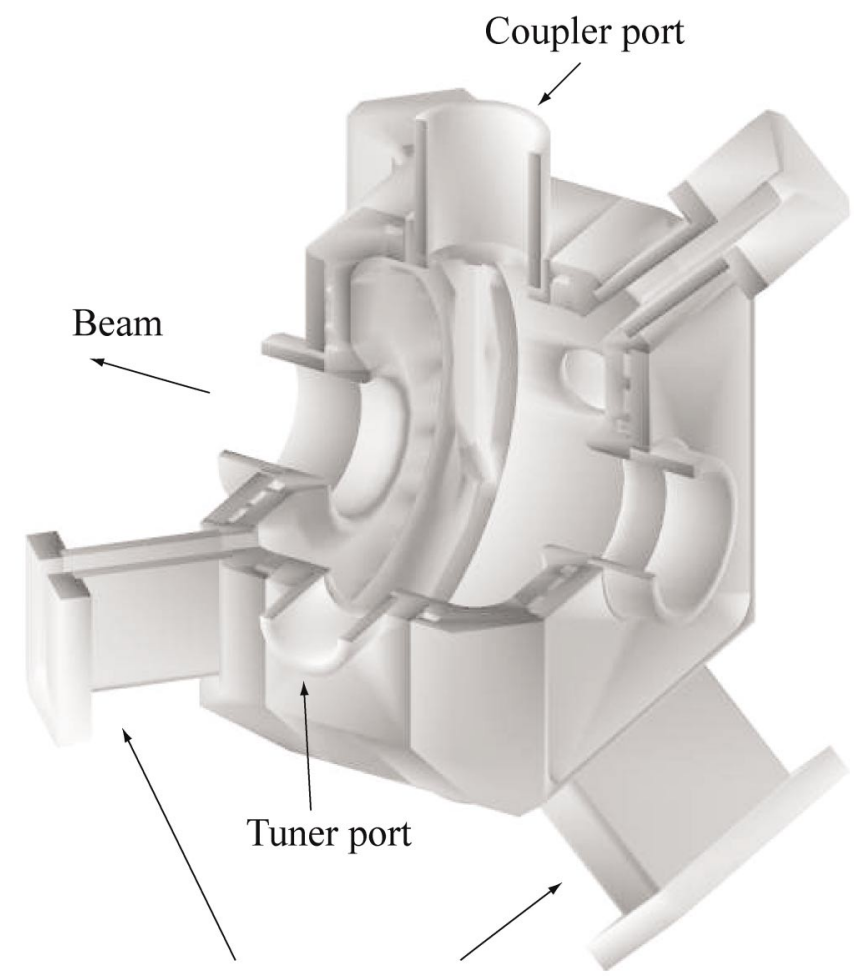

Waveguide ports for damping HOMs

FIG. 1. 714-MHz damped-accelerating cavity for the ATF damping ring, where a quarter of the cavity is cut out. It is equipped with four waveguide ports for damping higher-order modes. The ends of the HOM ports are to be terminated by broadband dummy loads, which are not shown here. 
is illustrated with some examples. We then discuss an application to cavity perturbation problems, where the splitting and mixing of eigenmodes can be understood clearly. We also discuss how the higher-order modes in the 714-MHz damped cavity can be classified.

\section{REPRESENTATION OF SYMMETRY OPERATIONS}

We consider an ideal rf cavity having a volume $V$, which is surrounded by a closed surface (or surfaces) $S$. A complete set of orthonormal functions can be derived from the following vector eigenvalue problem $[10,11]$ :

$$
\nabla^{2} \mathbf{E}_{n}+k_{n}^{2} \mathbf{E}_{n}=0, \quad(\text { in } V)
$$

and

$$
\mathbf{n} \times \mathbf{E}_{n}=0, \quad \nabla \cdot \mathbf{E}_{n}=0, \quad(\text { on } S)
$$

where $\mathbf{n}$ is an outer normal vector on $S$. The eigenvalues $k_{n}^{2}$ take discrete, real, and nonnegative values. Any vector function $\mathbf{A}$, which is piecewise continuous and square integrable over $V$, can be expanded in terms of the $\mathbf{E}_{n}$ 's. We assume that all eigenmodes $\mathbf{E}_{n}$ are real and that there are $d_{n}$ eigenmodes, $\mathbf{E}_{1}^{(n)}, \ldots, \mathbf{E}_{d_{n}}^{(n)}$, that belong to the same eigenvalue $k_{n}^{2}$.

Let us assume that the boundary surface $S$ is symmetric under some operation $R$ which brings the cavity into itself. For finite cavities, all distance-preserving operations can be constructed from rotations $(C)$ and space inversion $(I)$. We associate with $R$ a linear transformation $O_{R}$ for any vector function $\mathbf{E}(\mathbf{r})$. Concretely, corresponding to rotation $C$ that transforms a point $\mathbf{r}$ into $C \mathbf{r}$, the transformation $O_{C}$ is defined by

$$
O_{C} \mathbf{E}(\mathbf{r}) \equiv C \mathbf{E}\left(C^{-1} \mathbf{r}\right)
$$

which means that taking the vector $\mathbf{E}$ at a point $C^{-1} \mathbf{r}$, followed by a rotation of the vector by $C$, results in the vector $O_{C} \mathbf{E}$ at $\mathbf{r}$. For the other inversion $I$, which takes a point $\mathbf{r}$ into $-\mathbf{r}$, the corresponding transformation is defined by

$$
O_{I} \mathbf{E}(\mathbf{r}) \equiv-\mathbf{E}(-\mathbf{r})
$$

A set of such symmetry operations for the specified cavity forms a group. We shall call it the symmetry group of the cavity. To simplify the notations, we sometimes refer to $O_{R}$ as $R$.

Suppose that an eigenmode $\mathbf{E}_{m}^{(n)}$ is transformed by one of the operations $R$ of the symmetry group $G$. The transformed function satisfies Eqs. (1) and (2) with the same eigenvalue $k_{n}^{2}$ as the $\mathbf{E}_{m}^{(n)}$. Because of the completeness of the eigenmodes, it can be expressed as a linear combination of $d_{n}$ functions,

$$
O_{R} \mathbf{E}_{m}^{(n)}=\sum_{j=1}^{d_{n}} \mathbf{E}_{j}^{(n)} D_{j m}^{(n)}(R) .
$$

Then, we define a matrix $\hat{D}^{(n)}(R)$ by $\left[\hat{D}^{(n)}(R)\right]_{j m} \equiv$ $D_{j m}^{(n)}(R)$. Corresponding to a relation between the symmetry operations,

$$
R_{i} R_{j}=R_{k}
$$

the matrices satisfy the relation

$$
\hat{D}^{(n)}\left(R_{i}\right) \hat{D}^{(n)}\left(R_{j}\right)=\hat{D}^{(n)}\left(R_{k}\right) .
$$

Hence, the set of matrices $\hat{D}^{(n)}(R)$ for all $R \in G$ forms a representation of the symmetry group $G$. The $d_{n}$ eigenmodes $\left(\mathbf{E}_{j}^{(n)}\right)$ form a basis for this representation. Note that the matrices $\hat{D}^{(n)}(R)$ are orthogonal.

According to group theory [3-5], every representation can be decomposed into a direct sum of irreducible representations by rearranging basis functions properly by means of a linear transformation. Each basis function, rearranged in this way, belongs to an irreducible invariant subspace that is closed within itself under the operations of a symmetry group. In the present case, properly rearranged eigenmodes, $\mathbf{E}_{j}^{(n)}$, can be classified into the irreducible invariant subspaces, or into the irreducible representations, of the symmetry group $G$. Note that the above-obtained representation $\hat{D}^{(n)}(R)$ is irreducible in most cases; however, there are some exceptions, as illustrated in Sec. III B.

When the cavity has the symmetry group $G$, we start by finding irreducible representations of $G$. We can find them in the literature for well-known groups. The properly arranged eigenmodes can then be classified into the irreducible representations of the group $G$. Once the eigenmodes have been classified in this way, the symmetry of each eigenmode can be expressed by the corresponding representation. For example, if the eigenmodes $\mathbf{E}_{m}^{(\alpha)}(m=$ $1, \ldots, d_{\alpha}$ ) belong to an irreducible representation $\alpha$ of dimension $d_{\alpha}$ and have the same eigenvalue, they are transformed by an operation $R$ as

$$
O_{R} \mathbf{E}_{m}^{(\alpha)}=\sum_{j=1}^{d_{\alpha}} \mathbf{E}_{j}^{(\alpha)} D_{j m}^{(\alpha)}(R),
$$

where $D_{j m}^{(\alpha)}(R)$ is the $j m$ element of the $\alpha$-representation matrix for $R$.

If all symmetry operations commute, all irreducible representations are one dimensional. Then, each eigenmode can be chosen as a simultaneous eigenfunction for all symmetry operations. 
TABLE I. Character table for the point group $\mathrm{D}_{\infty \mathrm{h}}[3,4]$.

\begin{tabular}{l|cccccc}
\hline \hline Irreducible representation & $E$ & $C(\alpha)$ & $\sigma_{\mathrm{v}}$ & $I$ & $I C(\alpha)$ & $I \sigma_{\mathrm{v}}$ \\
\hline $\mathrm{A}_{1 \mathrm{~g}}$ & 1 & 1 & 1 & 1 & 1 & 1 \\
$\mathrm{~A}_{1 \mathrm{u}}$ & 1 & 1 & -1 & -1 & -1 & 1 \\
$\mathrm{~A}_{2 \mathrm{~g}}$ & 1 & 1 & -1 & 1 & 1 & -1 \\
$\mathrm{~A}_{2 \mathrm{u}}$ & 1 & 1 & 1 & -1 & -1 & -1 \\
$\mathrm{E}_{1 \mathrm{~g}}$ & 2 & $2 \cos \alpha$ & 0 & 2 & $2 \cos \alpha$ & 0 \\
$\mathrm{E}_{1 \mathrm{u}}$ & 2 & $2 \cos \alpha$ & 0 & -2 & $-2 \cos \alpha$ & 0 \\
$\mathrm{E}_{2 \mathrm{~g}}$ & 2 & $2 \cos 2 \alpha$ & 0 & 2 & $2 \cos 2 \alpha$ & 0 \\
$\mathrm{E}_{2 \mathrm{u}}$ & 2 & $2 \cos 2 \alpha$ & 0 & -2 & $-2 \cos 2 \alpha$ & 0 \\
$\vdots$ & $\vdots$ & $\vdots$ & $\vdots$ & $\vdots$ & $\vdots$ & $\vdots$ \\
$\mathrm{E}_{n \mathrm{~g}}$ & 2 & $2 \cos n \alpha$ & 0 & 2 & $2 \cos n \alpha$ & 0 \\
$\mathrm{E}_{n \mathrm{u}}$ & 2 & $2 \cos n \alpha$ & 0 & $-2-2 \cos n \alpha$ & 0 \\
\hline \hline
\end{tabular}

\section{EXAMPLES OF CLASSIFICATION}

\section{A. Axially symmetric cavities}

First, we consider a cylindrical pillbox cavity of radius $a$ and length $d$. We set the origin of the coordinate at the center of the cavity, and take the cavity axis as the $z$ axis. The cavity is symmetric about the group of symmetry operations $\mathrm{D}_{\infty \mathrm{h}} \equiv\left\{E, C(\alpha), \sigma_{\mathrm{v}}, I, I C(\alpha), I \sigma_{\mathrm{v}}\right\}$, where $E$ is the identity operation, $C(\alpha)$ is the rotation through an angle $\alpha$ about the $z$ axis, $\sigma_{\mathrm{v}}$ is the mirror reflection in a plane (sometimes referred to as the "vertical" plane) containing the $z$ axis. Note that there are an infinite number of rotations and reflections.

The irreducible representations of the group $\mathrm{D}_{\infty \mathrm{h}}$ are given in Table I. This table gives the characters corresponding to each operation and to each irreducible representation; the character is defined by the trace of a representation matrix. The group $D_{\infty h}$ has four onedimensional representations, named $\mathrm{A}_{1 \mathrm{~g}}, \mathrm{~A}_{1 \mathrm{u}}, \mathrm{A}_{2 \mathrm{~g}}$, and $\mathrm{A}_{2 \mathrm{u}}$, and infinite two-dimensional representations $\mathrm{E}_{n \mathrm{~g}}$ and $\mathrm{E}_{n \mathrm{u}}$, where the suffix " $\mathrm{g}$ " and " $\mathrm{u}$ " indicate even and odd parity under inversion, respectively, and $n$ is a positive integer indicating an azimuthal index. Note that each character for the one-dimensional representations indicates a representation matrix. For the other two-dimensional representations, typical representation matrices are given in Table II, for reference.

The eigenmodes in the pillbox cavity are given by the $\mathrm{TE}_{n m p}$ and $\mathrm{TM}_{n m p}$ modes, where $n, m$, and $p$ are integers. Examining how the electric-field modes transform under the symmetry operations, these modes can be classified
TABLE III. Classification of the eigenmodes in a pillbox cavity according to the irreducible representations of the group $\mathrm{D}_{\infty \mathrm{h}}$.

\begin{tabular}{lc}
\hline \hline Irreducible representation & Eigenmodes \\
\hline $\mathrm{A}_{1 \mathrm{~g}}$ & $\mathrm{TM}_{0 m p}(p:$ odd $)$ \\
$\mathrm{A}_{1 \mathrm{u}}$ & $\mathrm{TE}_{0 m p}(p:$ even $)$ \\
$\mathrm{A}_{2 \mathrm{~g}}$ & $\mathrm{TE}_{0 m p}(p:$ odd $)$ \\
$\mathrm{A}_{2 \mathrm{u}}$ & $\mathrm{TM}_{0 m p}(p:$ even $)$ \\
$\mathrm{E}_{n \mathrm{~g}}(n \geq 1)$ & $\mathrm{TE}_{n m p}, \mathrm{TM}_{n m p}(n+p:$ odd $)$ \\
$\mathrm{E}_{n \mathrm{u}}(n \geq 1)$ & $\mathrm{TE}_{n m p}, \mathrm{TM}_{n m p}(n+p:$ even $)$ \\
\hline \hline
\end{tabular}

according to the irreducible representations, as given in Table III. The classification of the magnetic-field modes yields the same result, assuming that the magnetic-field modes transform as axial vectors under inversion (see appendix).

Next, we consider a general cylindrical cavity that is axially symmetric about the $z$ axis, as well as mirror symmetric about the $x-y$ plane. Because this cavity has the same symmetry as the pillbox cavity, the eigenmodes can be classified similarly. As a result, $\mathrm{TE}_{0 m p}$-like modes and $\mathrm{TM}_{0 m p}$-like modes are classified into different representations. This indicates that the $\mathrm{TE}_{0 m p}$-like and $\mathrm{TM}_{0 m p}$-like modes are orthogonal to each other. On the other hand, both the $\mathrm{TE}_{n m p}$-like and $\mathrm{TM}_{n m p}$-like modes $(n \geq 1)$ belong to the same irreducible representation $\left(\mathrm{E}_{n \mathrm{~g}}\right.$ or $\mathrm{E}_{n \mathrm{u}}$ ) for the same $n$, which means that these modes are generally hybrid.

\section{B. Rectangular cavity}

We next consider a rectangular cavity of width $a$, height $b$, and length $d$. It provides an example of degeneracy that is specific to the vector eigenvalue problem. We take the coordinate system as shown in Fig. 2. This cavity has the symmetry group, $\mathrm{D}_{2 \mathrm{~h}} \equiv\left\{E, C_{2}^{z}, C_{2}^{y}, C_{2}^{x}, I, \sigma_{z}, \sigma_{y}, \sigma_{x}\right\}$, where $C_{2}^{z}, C_{2}^{y}$, and $C_{2}^{x}$ are the rotations through $\pi$ about the $z^{-}, y-$, and $x$ axes, respectively, and $\sigma_{z}, \sigma_{y}$, and $\sigma_{x}$ are mirror reflections in the $x-y, z-x$, and $y-z$ planes, respectively. The characters for the group $\mathrm{D}_{2 \mathrm{~h}}$ are given in Table IV. Because all symmetry operations commute, every irreducible representation is one dimensional.

The eigenmodes [12] in this cavity are specified by a set of integers $(n, m, p)$, where two of them must be different from zero. The corresponding eigenvalue is given by

TABLE II. Typical matrices of the representations, $\mathrm{E}_{n \mathrm{~g}}$ and $\mathrm{E}_{n \mathrm{u}}$, of the group $\mathrm{D}_{\infty \mathrm{h}}$. Note that a mirror reflection $\left(\sigma_{y}\right)$ about the $z-x$ plane has been chosen as a representative of the mirror reflections, $\sigma_{\mathrm{v}}$.

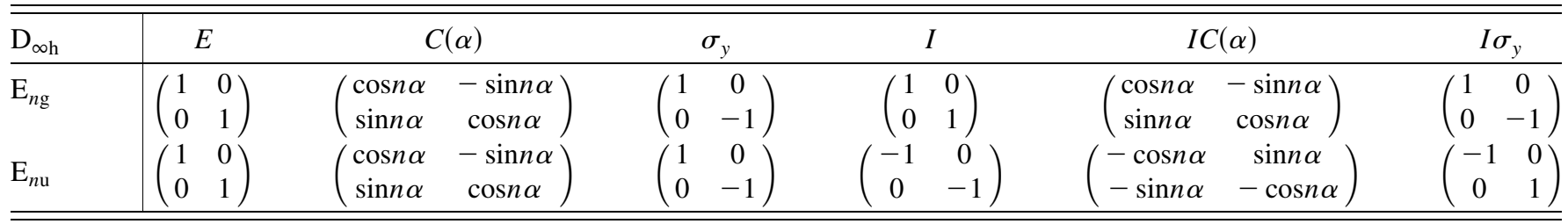




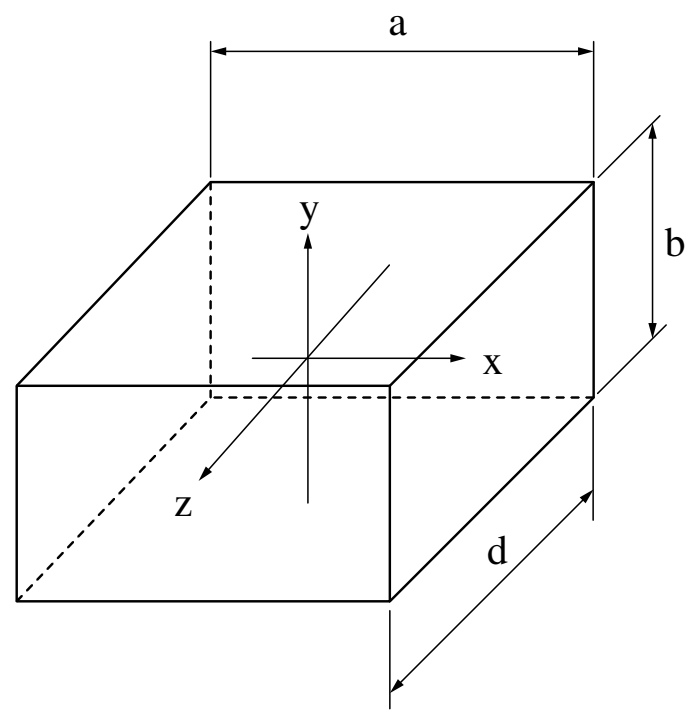

FIG. 2. Rectangular cavity.

$$
k_{n m p}^{2}=\left(\frac{n \pi}{a}\right)^{2}+\left(\frac{m \pi}{b}\right)^{2}+\left(\frac{p \pi}{d}\right)^{2} .
$$

Table $\mathrm{V}$ gives the classification of the eigenmodes according to the representations of $\mathrm{D}_{2 \mathrm{~h}}$. When all of the integers $(n, m, p)$ are different from zero, there are two independent eigenmodes corresponding to two possible polarizations. These degenerate modes belong to the same onedimensional representation. This situation contrasts with the cases of electronic states in quantum mechanics (without considering spin), where each one-dimensional representation contains only a single eigenfunction. The degeneracy in the rectangular cavity does not arise from the geometric symmetry, but from the freedom of polarization.

If we form a representation of the symmetry group by taking the above-mentioned degenerate modes corresponding to a set of $(n, m, p)$, we obtain a two-dimensional representation that is reducible into two one-dimensional representations. This is a typical example that the representation $\hat{D}^{(n)}(R)$ in Eq. (5) is reducible.

TABLE IV. Character table for the point group $\mathrm{D}_{2 \mathrm{~h}}$ [3].

\begin{tabular}{l|rrrrrrrr}
\hline \hline $\mathrm{D}_{2 \mathrm{~h}}$ & $E$ & $C_{2}^{z}$ & $C_{2}^{y}$ & $C_{2}^{x}$ & $I$ & $\sigma_{z}$ & $\sigma_{y}$ & $\sigma_{x}$ \\
\hline $\mathrm{A}_{\mathrm{g}}$ & 1 & 1 & 1 & 1 & 1 & 1 & 1 & 1 \\
$\mathrm{~B}_{1 \mathrm{~g}}$ & 1 & 1 & -1 & -1 & 1 & 1 & -1 & -1 \\
$\mathrm{~B}_{2 \mathrm{~g}}$ & 1 & -1 & 1 & -1 & 1 & -1 & 1 & -1 \\
$\mathrm{~B}_{3 \mathrm{~g}}$ & 1 & -1 & -1 & 1 & 1 & -1 & -1 & 1 \\
$\mathrm{~A}_{\mathrm{u}}$ & 1 & 1 & 1 & 1 & -1 & -1 & -1 & -1 \\
$\mathrm{~B}_{1 \mathrm{u}}$ & 1 & 1 & -1 & -1 & -1 & -1 & 1 & 1 \\
$\mathrm{~B}_{2 \mathrm{u}}$ & 1 & -1 & 1 & -1 & -1 & 1 & -1 & 1 \\
$\mathrm{~B}_{3 \mathrm{u}}$ & 1 & -1 & -1 & 1 & -1 & 1 & 1 & -1 \\
\hline \hline
\end{tabular}

TABLE V. Classification of the eigenmodes in a rectangular cavity. Each eigenmode is specified by a set of integers $(n, m, p)$. The corresponding irreducible representation is determined whether these integers are even or odd.

\begin{tabular}{l|ccc}
\hline \hline $\mathrm{D}_{2 \mathrm{~h}}$ & $n$ & $m$ & $p$ \\
\hline $\mathrm{A}_{\mathrm{g}}$ & Odd & Odd & Odd \\
$\mathrm{B}_{1 \mathrm{~g}}$ & Even & Even & Odd \\
$\mathrm{B}_{2 \mathrm{~g}}$ & Even & Odd & Even \\
$\mathrm{B}_{3 \mathrm{~g}}$ & Odd & Even & Even \\
$\mathrm{A}_{\mathrm{u}}$ & Even & Even & Even \\
$\mathrm{B}_{1 \mathrm{u}}$ & Odd & Odd & Even \\
$\mathrm{B}_{2 \mathrm{u}}$ & Odd & Even & Odd \\
$\mathrm{B}_{3 \mathrm{u}}$ & Even & Odd & Odd \\
\hline \hline
\end{tabular}

\section{APPLICATION TO PERTURBATION PROBLEMS}

\section{A. Handling perturbations with group theory}

When some perturbing object, such as a tuning plunger, is attached, it generally reduces the symmetry of the cavity. The perturbation can split degenerate modes and, furthermore, can couple those eigenmodes having close eigenvalues. Such behavior can be predicted qualitatively using group theory in a similar manner to that used in materials science [3].

The symmetry group $G^{\prime}$ for a perturbed cavity is a subgroup of the symmetry group $G$ for an original cavity. An irreducible representation $\Gamma_{0}$ of the group $G$ is, in general, reducible as a representation of $G^{\prime}$. If the $\Gamma_{0}$ can be decomposed into irreducible representations of $G^{\prime}$ as

$$
\Gamma_{0} \rightarrow \Gamma_{\alpha}+\Gamma_{\beta}+\cdots,
$$

original eigenmodes belonging to $\Gamma_{0}$ will be split into different eigenvalues corresponding to the representations of $\Gamma_{\alpha}, \Gamma_{\beta}, \ldots$, respectively. Such restriction of the representation $\Gamma_{0}$ to the subgroup $G^{\prime}$ is called a subduced representation, and is denoted by $\Gamma_{0} \downarrow G^{\prime}$.

We further assume that there are $N$ eigenmodes, $\mathbf{E}_{1}, \ldots, \mathbf{E}_{N}$, having close eigenvalues, and that the perturbation is relatively strong. Then, these modes can be coupled together under a suitable condition. The resulting coupled (mixed) modes can be approximately given by a linear combination,

$$
\tilde{\mathbf{E}}_{i}=\sum_{j=1}^{N} \mathbf{E}_{j} C_{j i}, \quad(i=1, \ldots, N)
$$

where $C_{j i}$ are the coefficients. We require that one of the new eigenmodes, $\tilde{\mathbf{E}}_{i}$, must belong to one of the irreducible representations $\Gamma_{\alpha}$ of the group $G^{\prime}$. Clearly, both sides of Eq. (11) must be transformed in the same way by any symmetry operation of $G^{\prime}$. This requires that each $\mathbf{E}_{j}$ on the right hand side must contain the representation of $\Gamma_{\alpha}$; otherwise, the coefficient $C_{j i}$ must be zero. In other words, 
in order for the original modes to be coupled together, they should contain a common irreducible representation of the group $G^{\prime}$. Some examples are presented in Sec. IV B.

It is worth noting that in the cases where the cavity boundary is slightly deformed from the volume $V$ (surface $S$ ) to $V^{\prime}$ $\left(S^{\prime}\right)$, the above-mentioned coefficients $C_{j i}$ can be determined so as to minimize the variational expression [11],

$$
k^{2}\left(\tilde{\mathbf{E}}_{i}\right)=\frac{\int_{V^{\prime}}\left[\left(\nabla \times \tilde{\mathbf{E}}_{i}\right)^{2}+\left(\nabla \cdot \tilde{\mathbf{E}}_{i}\right)^{2}\right] d v-2 \int_{S^{\prime}} \mathbf{n} \times \tilde{\mathbf{E}}_{i} \cdot \nabla \times \tilde{\mathbf{E}}_{i} d S}{\int_{V^{\prime}} \tilde{\mathbf{E}}_{i}^{2} d v} .
$$

\section{B. Examples of perturbations on the cavities}

As a first example, we consider a cylindrical pillbox cavity with two metallic objects attached to it, as shown in Fig. 3. The original cavity has the symmetry of $\mathrm{D}_{\infty \mathrm{h}}$. Because of the perturbation, the symmetry is reduced to $\mathrm{C}_{2 \mathrm{~h}}^{(y)} \equiv\left\{E, C_{2}^{y}, \sigma_{y}, I\right\}$, where the superscript $(y)$ denotes the twofold axis. The group $\mathrm{C}_{2 \mathrm{~h}}^{(y)}$ has four one-dimensional representations, as given in Table VI.

To predict the effect of a perturbation, we reduce each representation of $\mathrm{D}_{\infty \mathrm{h}}$ to a direct sum of irreducible representations of $C_{2 h}^{(y)}$. First, we select from Tables I and II the representation matrices of $\mathrm{D}_{\infty \mathrm{h}}$ corresponding to the elements of $\mathrm{C}_{2 \mathrm{~h}}^{(y)}$. The result is given in Table VII. We next calculate the characters of them, yielding Table VIII. When a representation $D$ is a direct sum of irreducible representations $D^{(\alpha)}$,

$$
D=\sum_{\alpha} q_{\alpha} D^{(\alpha)},
$$

where $q_{\alpha}$ are nonnegative integers, the $q_{\alpha}$ can be determined by a relation [3],

$$
q_{\alpha}=\frac{1}{g} \sum_{R} \chi^{(\alpha)}(R)^{*} \chi(R),
$$

where $R$ is the element of the group, $g$ is the number of elements, $\chi(R)$ and $\chi^{(\alpha)}(R)$ are the characters of the representations $D(R)$ and $D^{(\alpha)}(R)$, respectively, and the asterisk denotes the complex conjugate. Using Eq. (14),
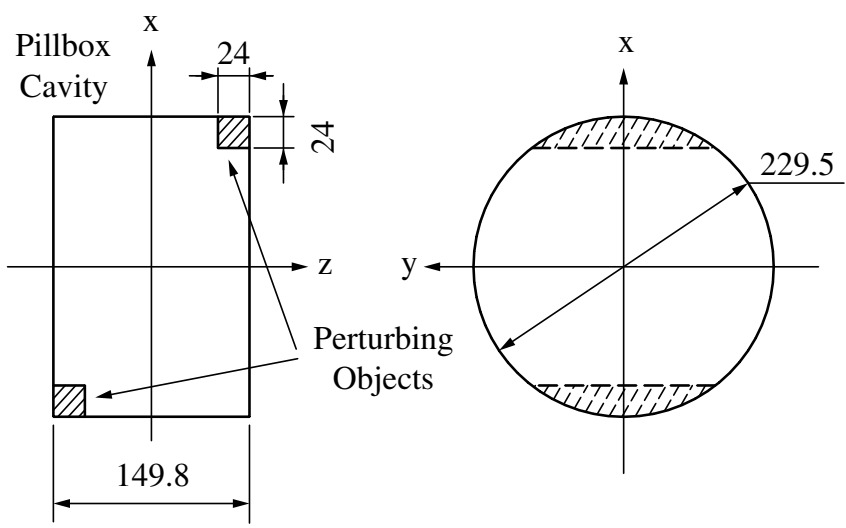

FIG. 3. Pillbox cavity (fundamental frequency: $1 \mathrm{GHz}$ ) with two metallic objects. Units: $\mathrm{mm}$. each representation of $\mathrm{D}_{\infty \mathrm{h}}$ is decomposed as shown in Table IX. For example, two-dimensional representations, $\mathrm{E}_{n \mathrm{~g}}$ and $\mathrm{E}_{n \mathrm{u}}$, are decomposed as $\mathrm{E}_{n \mathrm{~g}} \downarrow \mathrm{C}_{2 \mathrm{~h}}^{(y)}=\mathrm{A}_{\mathrm{g}}+\mathrm{B}_{\mathrm{g}}$ and $\mathrm{E}_{n \mathrm{u}} \downarrow \mathrm{C}_{2 \mathrm{~h}}^{(y)}=\mathrm{A}_{\mathrm{u}}+\mathrm{B}_{\mathrm{u}}$, respectively. This indicates that a twofold degeneracy in both $\mathrm{E}_{n \mathrm{~g}}$ and $\mathrm{E}_{n \mathrm{u}}$ will be split into two levels (eigenvalues).

Figure 4 shows the eigenfrequencies for the original pillbox cavity as well as those for the perturbed cavity. These results were calculated using the computer code

TABLE VI. Character table for the point group $C_{2 h}^{(y)}$ [4]. The superscript $(y)$ denotes that a twofold axis is the $y$ axis.

\begin{tabular}{l|lrrr}
\hline \hline $\mathrm{C}_{2 \mathrm{~h}}^{(y)}$ & $E$ & $C_{2}^{y}$ & $\sigma_{y}$ & $I$ \\
\hline $\mathrm{A}_{\mathrm{g}}$ & 1 & 1 & 1 & 1 \\
$\mathrm{~A}_{\mathrm{u}}$ & 1 & 1 & -1 & -1 \\
$\mathrm{~B}_{\mathrm{g}}$ & 1 & -1 & -1 & 1 \\
$\mathrm{~B}_{\mathrm{u}}$ & 1 & -1 & 1 & -1 \\
\hline \hline
\end{tabular}

TABLE VII. Selected representation matrices of $\mathrm{D}_{\infty \mathrm{h}}$ for the elements of $\mathrm{C}_{2 \mathrm{~h}}^{(y)}$. The matrices for the operation $C_{2}^{y}$ were derived using a relation $C_{2}^{y}=I \sigma_{y}$.

\begin{tabular}{l|cccc}
\hline \hline $\mathrm{D}_{\infty \mathrm{h}}$ & $E$ & $C_{2}^{y}$ & $\sigma_{y}$ & $I$ \\
\hline $\mathrm{A}_{1 \mathrm{~g}}$ & 1 & 1 & 1 & 1 \\
$\mathrm{~A}_{1 \mathrm{u}}$ & 1 & 1 & -1 & -1 \\
$\mathrm{~A}_{2 \mathrm{~g}}$ & 1 & -1 & -1 & 1 \\
$\mathrm{~A}_{2 \mathrm{u}}$ & 1 & -1 & 1 & -1 \\
$\mathrm{E}_{n \mathrm{~g}}$ & $\left(\begin{array}{cc}1 & 0 \\
0 & 1\end{array}\right)$ & $\left(\begin{array}{cc}1 & 0 \\
0 & -1\end{array}\right)$ & $\left(\begin{array}{cc}1 & 0 \\
0 & -1\end{array}\right)$ & $\left(\begin{array}{cc}1 & 0 \\
0 & 1\end{array}\right)$ \\
$\mathrm{E}_{n \mathrm{u}}$ & $\left(\begin{array}{ll}1 & 0 \\
0 & 1\end{array}\right)$ & $\left(\begin{array}{cc}-1 & 0 \\
0 & 1\end{array}\right)$ & $\left(\begin{array}{cc}1 & 0 \\
0 & -1\end{array}\right)$ & $\left(\begin{array}{cc}-1 & 0 \\
0 & -1\end{array}\right)$ \\
\hline \hline
\end{tabular}

TABLE VIII. Characters of the representations of $\mathrm{D}_{\infty \mathrm{h}}$ for the elements of $\mathrm{C}_{2 \mathrm{~h}}^{(y)}$.

\begin{tabular}{l|lrrr}
\hline \hline $\mathrm{D}_{\infty \mathrm{h}}$ & $E$ & $C_{2}^{y}$ & $\sigma_{y}$ & $I$ \\
\hline $\mathrm{A}_{1 \mathrm{~g}}$ & 1 & 1 & 1 & 1 \\
$\mathrm{~A}_{1 \mathrm{u}}$ & 1 & 1 & -1 & -1 \\
$\mathrm{~A}_{2 \mathrm{~g}}$ & 1 & -1 & -1 & 1 \\
$\mathrm{~A}_{2 \mathrm{u}}$ & 1 & -1 & 1 & -1 \\
$\mathrm{E}_{n \mathrm{~g}}$ & 2 & 0 & 0 & 2 \\
$\mathrm{E}_{n \mathrm{u}}$ & 2 & 0 & 0 & -2 \\
\hline \hline
\end{tabular}


TABLE IX. Compatibility relations between the irreducible representations of $\mathrm{D}_{\infty \mathrm{h}}$ and $\mathrm{C}_{2 \mathrm{~h}}^{(y)}$.

\begin{tabular}{lc}
\hline \hline $\mathrm{D}_{\infty \mathrm{h}}$ & $\mathrm{C}_{2 \mathrm{~h}}^{(y)}$ \\
\hline $\mathrm{A}_{1 \mathrm{~g}}$ & $\mathrm{~A}_{\mathrm{g}}$ \\
$\mathrm{A}_{1 \mathrm{u}}$ & $\mathrm{A}_{\mathrm{u}}$ \\
$\mathrm{A}_{2 \mathrm{~g}}$ & $\mathrm{~B}_{\mathrm{g}}$ \\
$\mathrm{A}_{2 \mathrm{u}}$ & $\mathrm{B}_{\mathrm{u}}$ \\
$\mathrm{E}_{n \mathrm{~g}}$ & $\mathrm{~A}_{\mathrm{g}}+\mathrm{B}_{\mathrm{g}}$ \\
$\mathrm{E}_{n \mathrm{u}}$ & $\mathrm{A}_{\mathrm{u}}+\mathrm{B}_{\mathrm{u}}$ \\
\hline \hline
\end{tabular}

MAFIA [13]. We first look at the second lowest $\left(\mathrm{TE}_{111}\right)$ modes in the original cavity, which belong to twodimensional representation $\mathrm{E}_{1 \mathrm{u}}$. Under the perturbation, this level is split into two levels of $A_{u}$ and $B_{u}$, showing a typical mode-splitting behavior. A more complicated situation happens to the fourth $\left(\mathrm{TM}_{110}\right)$ and the fifth $\left(\mathrm{TE}_{211}\right)$ modes, each belonging to $E_{1 g}$ and $E_{2 g}$, respectively. Under the perturbation, each of them splits into the representations of $A_{g}+B_{g}$. Furthermore, because these modes have very close eigenfrequencies, two of the resulting modes belonging to $\mathrm{A}_{\mathrm{g}}$ are coupled together, while the other two belonging to $\mathrm{B}_{\mathrm{g}}$ are coupled as well. As a result, we obtain two pairs of mixed modes from the $\mathrm{TM}_{110}$ and $\mathrm{TE}_{211}$ modes.

As a second example, we consider another cavity shown in Fig. 5. This cavity has similar perturbing objects to those

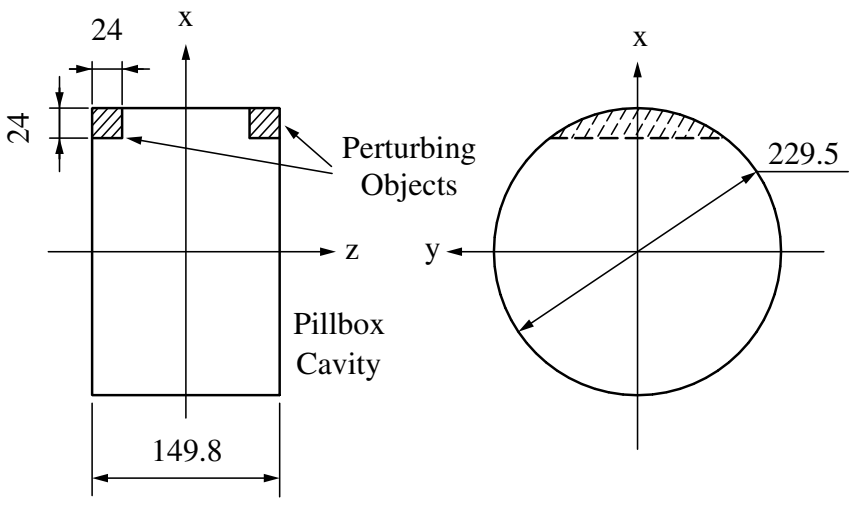

FIG. 5. Second example of a perturbed cavity. Two metallic objects are attached on one $(x>0)$ side of the pillbox cavity.

in Fig. 3, but both of them are placed on the same side of $x>0$. The perturbed cavity has the symmetry $\mathrm{C}_{2 \mathrm{v}}^{(x)} \equiv$ $\left\{E, C_{2}^{x}, \sigma_{z}, \sigma_{y}\right\}$, where the superscript $(x)$ denotes the twofold axis. The irreducible representations of $\mathrm{C}_{2 \mathrm{v}}^{(x)}$ are given in Table $\mathrm{X}$. The compatibility relations between the $\mathrm{D}_{\infty \mathrm{h}}$ and $\mathrm{C}_{2 \mathrm{v}}^{(x)}$ are given in Table XI.

The mode spectra for this cavity are shown in Fig. 6. We pay attention to the fourth $\left(\mathrm{TM}_{110}\right)$ and the fifth $\left(\mathrm{TE}_{211}\right)$ modes in the original cavity, again. In the present case, the representations $E_{1 \mathrm{~g}}$ and $\mathrm{E}_{2 \mathrm{~g}}$ are decomposed into $\mathrm{A}_{2}+\mathrm{B}_{2}$ and $A_{1}+B_{1}$, respectively. Because all of the resulting representations are different, there is no coupling between

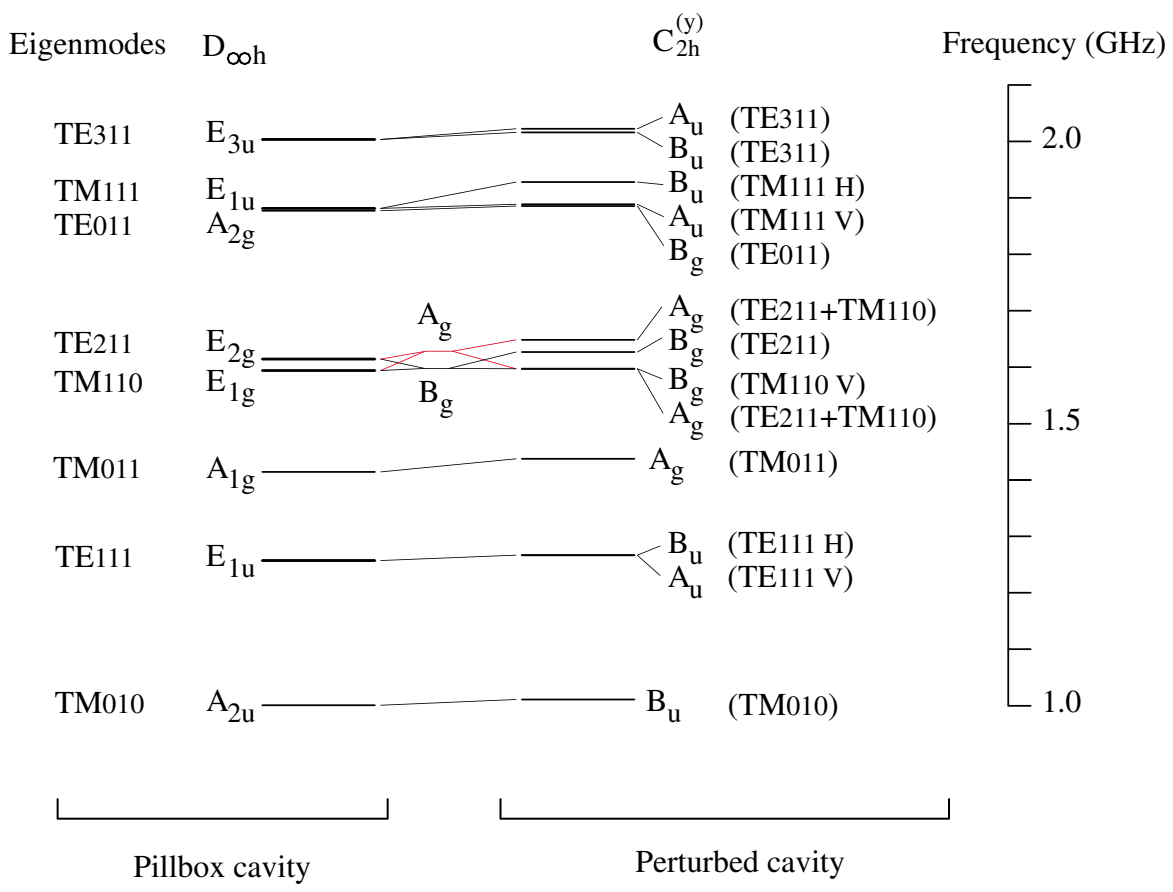

FIG. 4. (Color) Eigenmode spectra for the original pillbox cavity and for the perturbed cavity (in Fig. 3). Every eigenfrequency is indicated by a horizontal line, together with its classification according to the irreducible representation. Thick lines for the pillbox cavity indicate twofold degeneracy. 
TABLE $X$. Character table for the point group $C_{2 \mathrm{v}}^{(x)}[3,4]$. The superscript $(x)$ denotes that a twofold axis is the $x$ axis.

\begin{tabular}{l|lrrr}
\hline \hline $\mathrm{C}_{2 \mathrm{v}}^{(x)}$ & $E$ & $C_{2}^{x}$ & $\sigma_{z}$ & $\sigma_{y}$ \\
\hline $\mathrm{A}_{1}$ & 1 & 1 & 1 & 1 \\
$\mathrm{~A}_{2}$ & 1 & 1 & -1 & -1 \\
$\mathrm{~B}_{1}$ & 1 & -1 & 1 & -1 \\
$\mathrm{~B}_{2}$ & 1 & -1 & -1 & 1 \\
\hline \hline
\end{tabular}

TABLE XI. Compatibility relations between the irreducible representations of $\mathrm{D}_{\infty \mathrm{h}}$ and $\mathrm{C}_{2 \mathrm{v}}^{(x)}$.

\begin{tabular}{lc}
\hline \hline $\mathrm{D}_{\infty \mathrm{h}}$ & $\mathrm{C}_{2 \mathrm{v}}^{(x)}$ \\
\hline $\mathrm{A}_{1 \mathrm{~g}}$ & $\mathrm{~A}_{1}$ \\
$\mathrm{~A}_{1 \mathrm{u}}$ & $\mathrm{A}_{2}$ \\
$\mathrm{~A}_{2 \mathrm{~g}}$ & $\mathrm{~B}_{1}$ \\
$\mathrm{~A}_{2 \mathrm{u}}$ & $\mathrm{B}_{2}$ \\
$\mathrm{E}_{1 \mathrm{~g}}$ & $\mathrm{~A}_{2}+\mathrm{B}_{2}$ \\
$\mathrm{E}_{1 \mathrm{u}}$ & $\mathrm{A}_{1}+\mathrm{B}_{1}$ \\
$\mathrm{E}_{2 \mathrm{~g}}$ & $\mathrm{~A}_{1}+\mathrm{B}_{1}$ \\
$\mathrm{E}_{2 \mathrm{u}}$ & $\mathrm{A}_{2}+\mathrm{B}_{2}$ \\
$\vdots$ & $\vdots$ \\
\hline \hline
\end{tabular}

these modes. Then, both the $\mathrm{TM}_{110}$ and the $\mathrm{TE}_{211}$ modes merely split in their levels.

The above two examples demonstrate that the symmetry of the perturbed cavity determines whether the neighboring modes can be coupled through the perturbation.

\section{CLASSIFICATION OF EIGENMODES IN THE 714-MHZ DAMPED CAVITY}

In the 1.54-GeV ATF damping ring [14] at KEK, two 714-MHz accelerating cavities have been in operation since 1997. Harmful higher-order modes in these cavities can be heavily damped by using four dedicated HOM ports (170-mm wide by $20-\mathrm{mm}$ high each). While developing these cavities, the characteristics of HOMs were investigated [15] using a low-power test cavity having four demountable HOM ports. The HOMs were examined with and without attaching these ports, respectively, for a comparison. It was found that the field distributions of some HOMs in a port-removed cavity (see Fig. 7) were very different from those from two-dimensional $(r-z)$ calculations; these modes were largely influenced by the space left at the slots for the ports.

We classify here the eigenmodes in this port-removed cavity. The eigenmodes in a real damped cavity will be classified similarly because it has the same symmetry as the port-removed cavity if we neglect small effects of a tuning plunger and of an input coupler.

There are eight equivalent points for the port-removed cavity, as indicated in Fig. 8. This cavity is symmetric about the group $\mathrm{D}_{2 \mathrm{~d}} \equiv\left\{E, C_{2}, I C_{4}, I C_{4}^{-1}, C_{2}^{\prime}, C_{2}^{\prime \prime}, \sigma_{x}, \sigma_{y}\right\}$, where $C_{2}$ and $C_{4}$ are the rotations about the $z$ axis, and $C_{2}^{\prime}$ and $C_{2}^{\prime \prime}$ are the rotations through $\pi$ about the [110] and [110] axes, respectively. A character table for the group $\mathrm{D}_{2 \mathrm{~d}}$ is given in Table XII. This group has four 1dimensional representations and one 2-dimensional representation.

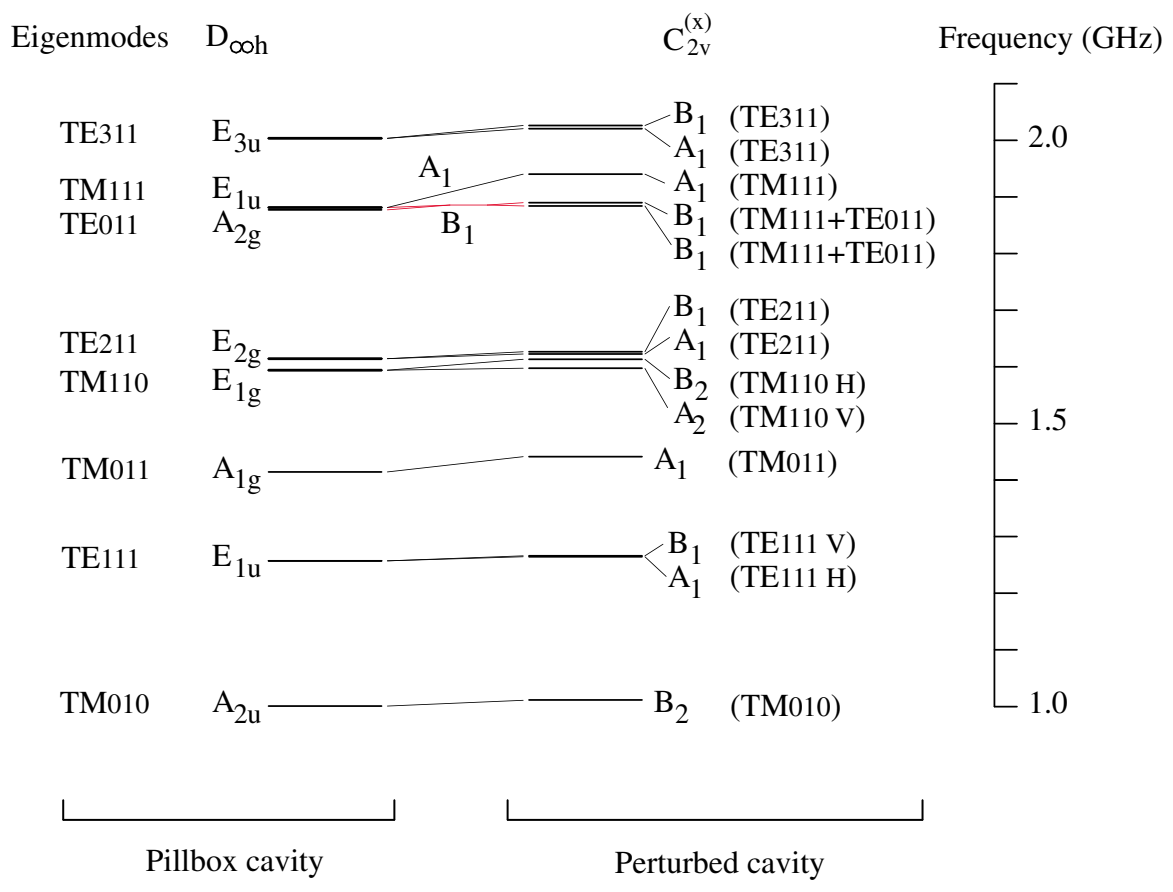

FIG. 6. (Color) Calculated mode spectra for the cavity in Fig. 5, together with those for the original pillbox cavity. The thick lines for the pillbox cavity indicate twofold degeneracy. 


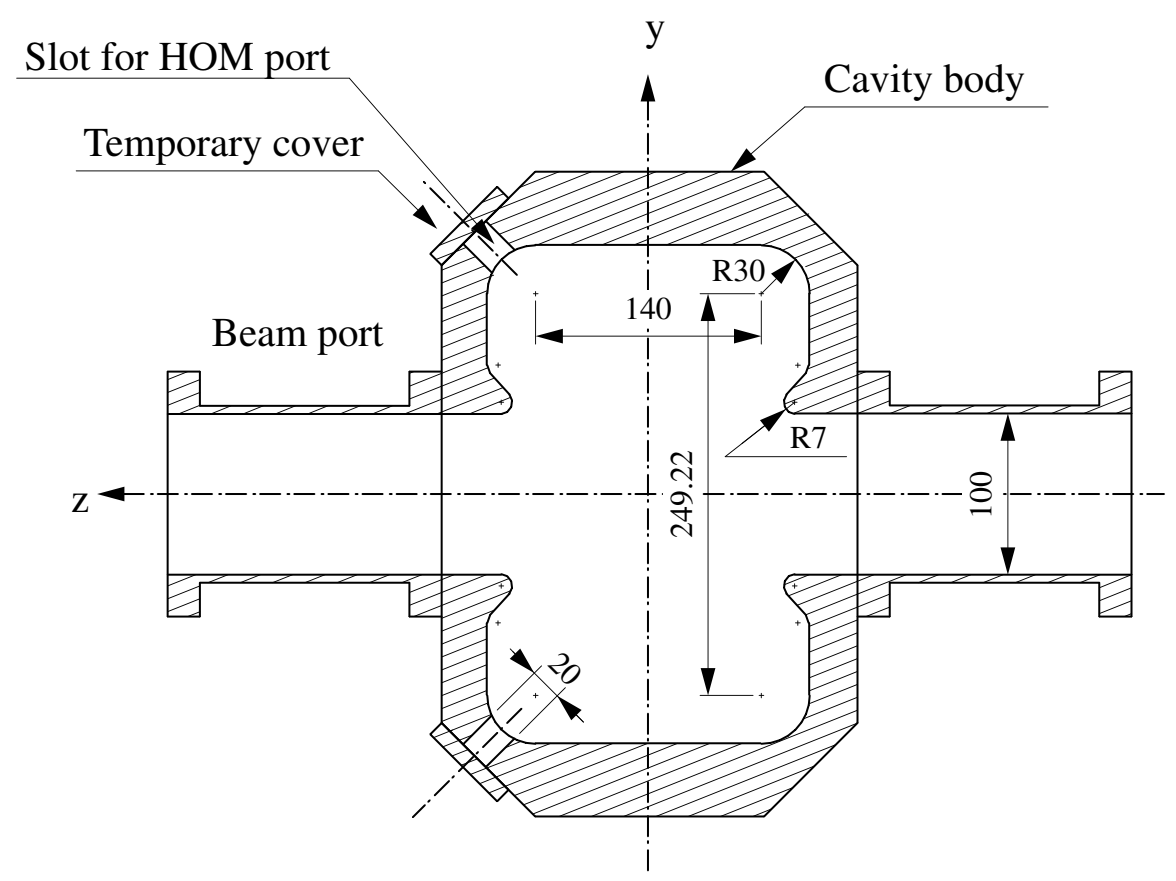

FIG. 7. Cross section of a low-power model of the 714-MHz damped cavity, where HOM ports have been removed and shorted. There are two other slots for HOM ports, which are located on the other $(z<0)$ side with $90^{\circ}$ rotation. Units: mm.

Figures 9 and 10 show the calculated spectra of the eigenmodes in the port-removed cavity, together with those in an unperturbed (axially symmetric) cavity. The correspondence of the modes between these cavities was found by examining their field distributions, while noting the compatibility relations (in Table XIII). The first figure [Fig. 9(a)] indicates that (i) each mode belonging to a one-dimensional representation merely shifts in frequency, and (ii) degenerate modes belonging to $\mathrm{E}_{2 \mathrm{~g}}$ split into two levels, $\mathrm{B}_{1}$ and $\mathrm{B}_{2}$.

The next figure [Fig. 9(b)] shows a more complicated behavior. The unperturbed cavity has four close levels

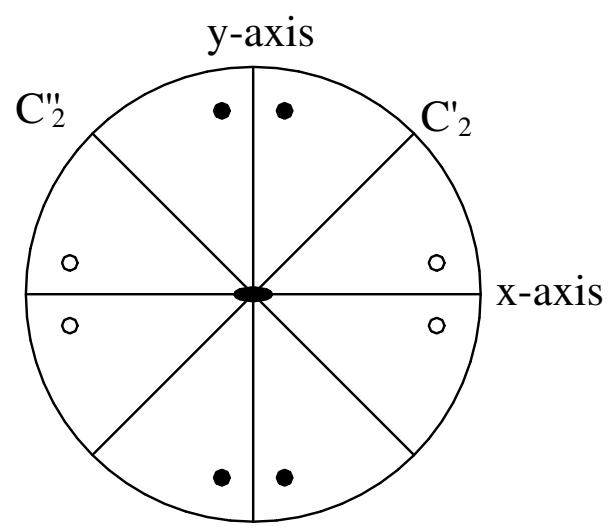

FIG. 8. Stereogram of the point group $\mathrm{D}_{2 \mathrm{~d}}$. The white and black circles indicate the points above and below the sheet, respectively. Note that the group $\mathrm{D}_{2 \mathrm{~d}}$ is usually indicated by a similar figure with $45^{\circ}$ rotation. (belonging to $A_{2 u}, E_{2 g}, E_{2 u}$, and $E_{4 g}$ ) in a frequency range of 1.74-1.81 GHz. Under the perturbation, these representations are reduced to $A_{2 \mathrm{u}} \downarrow \mathrm{D}_{2 \mathrm{~d}}=\mathrm{B}_{2}, \mathrm{E}_{2 \mathrm{~g}} \downarrow \mathrm{D}_{2 \mathrm{~d}}=\mathrm{B}_{1}+$ $\mathrm{B}_{2}, \mathrm{E}_{2 \mathrm{u}} \downarrow \mathrm{D}_{2 \mathrm{~d}}=\mathrm{A}_{1}+\mathrm{A}_{2}$, and $\mathrm{E}_{4 \mathrm{~g}} \downarrow \mathrm{D}_{2 \mathrm{~d}}=\mathrm{A}_{1}+\mathrm{A}_{2}$, respectively, which result in splittings of the degenerate levels. Furthermore, some of the split modes having the same representation can be coupled together. An inspection of the field distributions suggests that there are some couplings between the split modes, as indicated by the thin lines in Fig. 9(b).

In the third figure (Fig. 10), antisymmetric modes about the rotation $C_{2}$ are shown. The representations $\mathrm{E}_{n \mathrm{~g}}$ and $\mathrm{E}_{n \mathrm{u}}$ ( $n$ : odd) of $\mathrm{D}_{\infty \mathrm{h}}$ are compatible to two-dimensional representation $E$ of $D_{2 d}$. As a result, there is no splitting of the levels. Only two uppermost modes (1-E-3 and 1-M-3) are coupled together by the perturbation.

This example shows that the group theoretical method is very useful for classifying the eigenmodes in a complicated cavity, and for understanding the coupling between the eigenmodes.

TABLE XII. Character table for the point group $\mathrm{D}_{2 \mathrm{~d}}$ [3].

\begin{tabular}{l|ccrrr}
\hline \hline $\mathrm{D}_{2 \mathrm{~d}}$ & $E$ & $I C_{4}, I C_{4}^{-1}$ & $C_{2}$ & $C_{2}^{\prime}, C_{2}^{\prime \prime}$ & $\sigma_{x}, \sigma_{y}$ \\
\hline $\mathrm{A}_{1}$ & 1 & 1 & 1 & 1 & 1 \\
$\mathrm{~A}_{2}$ & 1 & 1 & 1 & -1 & -1 \\
$\mathrm{~B}_{1}$ & 1 & -1 & 1 & 1 & -1 \\
$\mathrm{~B}_{2}$ & 1 & -1 & 1 & -1 & 1 \\
$\mathrm{E}$ & 2 & 0 & -2 & 0 & 0 \\
\hline \hline
\end{tabular}




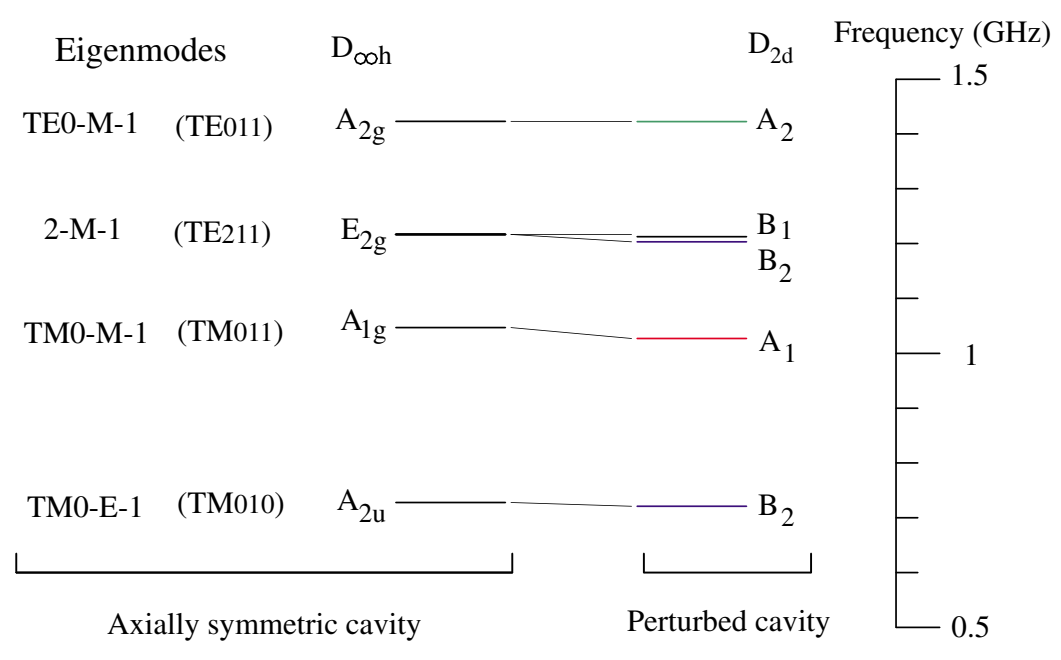

(a)

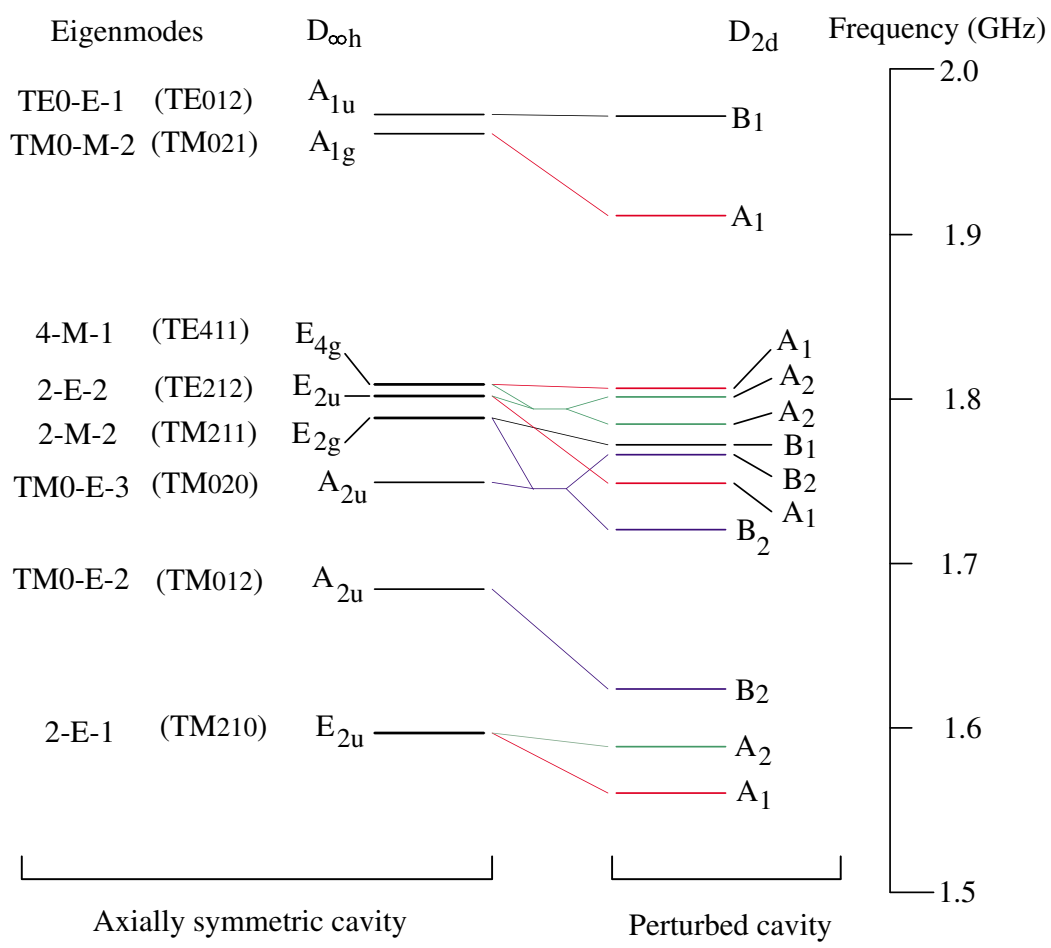

(b)

FIG. 9. (Color) Calculated spectra of eigenmodes for an axially symmetric cavity (without HOM ports) and for a perturbed cavity (with four slots; in Fig. 7). Symmetric modes about the rotation $C_{2}$ are shown. Frequency range: (a) $0.5-1.5 \mathrm{GHz}$, and (b) $1.5-2 \mathrm{GHz}$. The thick lines indicate twofold degenerate levels. Each eigenmode in the axially symmetric cavity is labeled by a set of an azimuthal index, a boundary condition (Magnetic or Electric short) on the $x-y$ plane, and a number; the corresponding mode in an ideal pillbox cavity is indicated in parentheses.

\section{CONCLUSIONS}

The geometric symmetry of a cavity can be expressed by the group of symmetry operations. By taking basis functions from a complete set of eigenmodes of the vector Helmholtz equation, we can form a representation of the symmetry group. The eigenmodes in the cavity can then be classified according to irreducible representations of the symmetry group. This method is particularly useful for classifying the eigenmodes in complicated cavities, and for understanding the behavior of the eigenmodes under perturbations. 


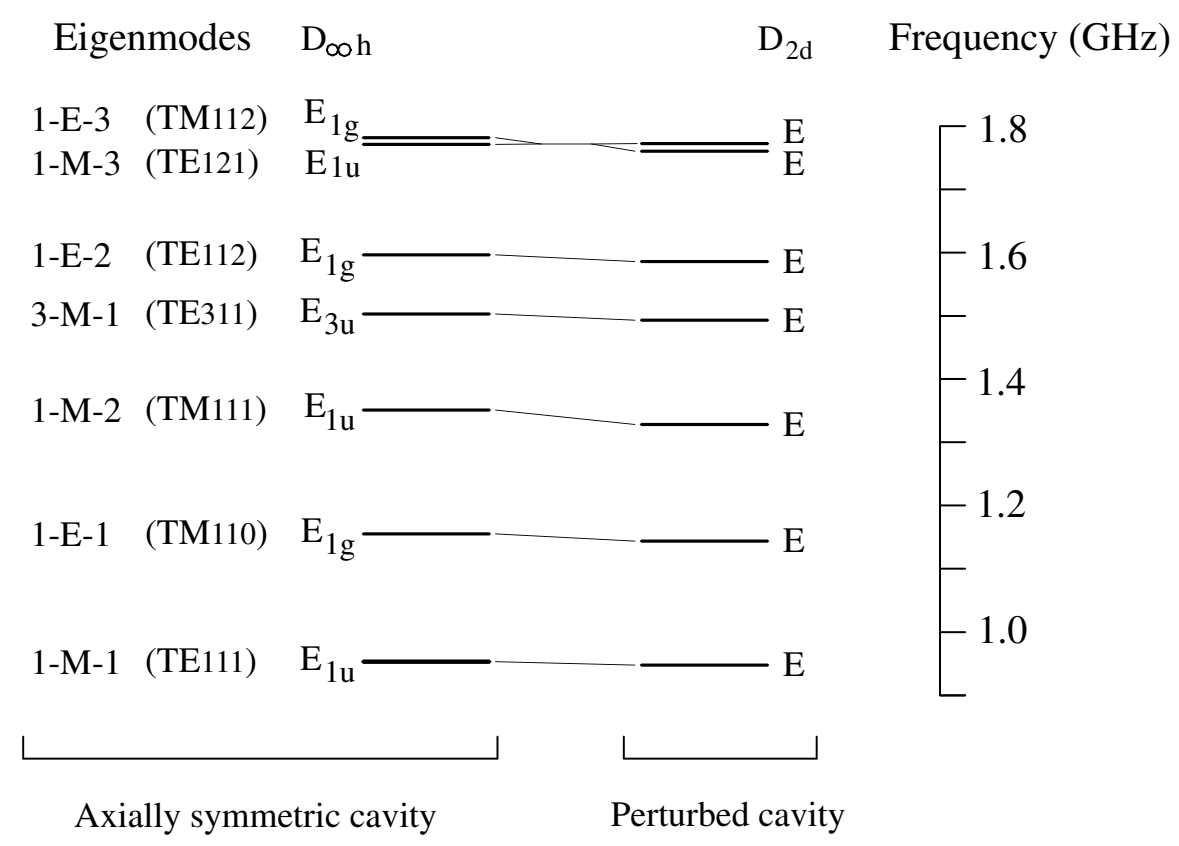

FIG. 10. Calculated spectra of eigenmodes that are antisymmetric about the rotation $C_{2}$. The frequency range is $0.9-1.8 \mathrm{GHz}$.

TABLE XIII. Compatibility relations between the irreducible representations of $\mathrm{D}_{\infty \mathrm{h}}$ and $\mathrm{D}_{2 \mathrm{~d}}$.

\begin{tabular}{lc}
\hline \hline$D_{\infty \mathrm{h}}$ & $\mathrm{D}_{2 \mathrm{~d}}$ \\
\hline $\mathrm{A}_{1 \mathrm{~g}}$ & $\mathrm{~A}_{1}$ \\
$\mathrm{~A}_{1 \mathrm{u}}$ & $\mathrm{B}_{1}$ \\
$\mathrm{~A}_{2 \mathrm{~g}}$ & $\mathrm{~A}_{2}$ \\
$\mathrm{~A}_{2 \mathrm{u}}$ & $\mathrm{B}_{2}$ \\
$\mathrm{E}_{1 \mathrm{~g}}$ & $\mathrm{E}$ \\
$\mathrm{E}_{1 \mathrm{u}}$ & $\mathrm{E}$ \\
$\mathrm{E}_{2 \mathrm{~g}}$ & $\mathrm{~B}_{1}+\mathrm{B}_{2}$ \\
$\mathrm{E}_{2 \mathrm{u}}$ & $\mathrm{A}_{1}+\mathrm{A}_{2}$ \\
$\mathrm{E}_{3 \mathrm{~g}}$ & $\mathrm{E}$ \\
$\mathrm{E}_{3 \mathrm{u}}$ & $\mathrm{E}$ \\
$\mathrm{E}_{4 \mathrm{~g}}$ & $\mathrm{~A}_{1}+\mathrm{A}_{2}$ \\
$\mathrm{E}_{4 \mathrm{u}}$ & $\mathrm{B}_{1}+\mathrm{B}_{2}$ \\
$\vdots$ & $\vdots$ \\
\hline \hline
\end{tabular}

\section{ACKNOWLEDGMENTS}

The author would like to thank Dr. M. Izawa, Dr. T. Koide, and Dr. H. Maezawa for useful discussions.

\section{APPENDIX: TRANSFORMATIONS OF MAGNETIC-FIELD MODES}

In Sec. II, we dealt with electric-field modes derived from Eqs. (1) and (2). Another complete set of eigenmodes, suitable for expanding magnetic fields, is derived from the eigenvalue problem $[10,11]$,

$$
\nabla^{2} \mathbf{H}_{n}+k_{n}^{2} \mathbf{H}_{n}=0, \quad(\text { in } V)
$$

with boundary conditions

$$
\mathbf{n} \cdot \mathbf{H}_{n}=0, \quad \mathbf{n} \times \nabla \times \mathbf{H}_{n}=0 \quad(\text { on } S) .
$$

Such eigenmodes shall be called magnetic-field modes. For solenoidal modes, there is a one-to-one correspondence between the electric- and magnetic-field modes, which is given by

$$
\nabla \times \mathbf{E}_{n}=k_{n} \mathbf{H}_{n}, \quad \nabla \times \mathbf{H}_{n}=k_{n} \mathbf{E}_{n} .
$$

If we assume the $\mathbf{E}_{n}$ 's as polar vectors, any solenoidal mode among the $\mathbf{H}_{n}$ 's must be an axial vector. Then, it is natural to assume all $\mathbf{H}_{n}$ 's are axial vectors. We assume that they are transformed by inversion, as

$$
O_{I} \mathbf{H}_{n}(\mathbf{r}) \equiv \mathbf{H}_{n}(-\mathbf{r})
$$

If we take a set of eigenmodes $\mathbf{E}_{m}^{(\alpha)}\left(m=1, \ldots, d_{\alpha}\right)$ belonging to an irreducible representation $\alpha$ of the symmetry group, and having the same eigenvalue $k_{n}$, they are transformed by any symmetry operation $R$ as given in Eq. (8). Using the relation

$$
O_{R}\left(\nabla \times \mathbf{E}_{n}\right)=\nabla \times\left(O_{R} \mathbf{E}_{n}\right),
$$

which is valid for any operation $R$, including both inversion and rotation, we can easily show that the magnetic-field modes $\mathbf{H}_{m}$ are transformed in the same way as the corresponding $\mathbf{E}_{m}^{(\alpha)}$. Thus, the corresponding electric- and magnetic-field modes are classified into the same irreducible representation, provided that they are solenoidal modes. 
[1] See, for example, R. Boni, in Proceedings of the Fifth European Particle Accelerator Conference, Sitges (Barcelona), 1996 (Institute of Physics Publishing, Bristol, U.K., 1996), pp. 182-186.

[2] S. Sakanaka, F. Hinode, K. Kubo, and J. Urakawa, J. Synchrotron Radiat. 5, 386 (1998).

[3] T. Inui, Y. Tanabe, and Y. Onodera, Group Theory and Its Applications in Physics (Springer-Verlag, Berlin, 1990).

[4] Morton Hamermesh, Group Theory and Its Application to Physical Problems (Dover, New York, 1989).

[5] Volker Heine, Group Theory in Quantum Mechanics (Dover, New York, 1993).

[6] R. H. Dicke, in Principles of Microwave Circuits, edited by C. G. Montgomery, R. H. Dicke, and E. M. Purcell (McGraw-Hill, New York, 1948), Chap. 12.

[7] Robert M. Bevensee, Ann. Phys. (N.Y.) 12, 222 (1961).

[8] E.M. Nelson, in Computational Accelerator Physics, edited by Robert Ryne, AIP Conf. Proc. No. 297 (American Institute of Physics, New York, 1994), pp. 323-332.

[9] See, for example, K. Sakoda, Phys. Rev. B 52, 7982 (1995); K. Ohtaka and Y. Tanabe, J. Phys. Soc. Jpn. 65, 2670 (1996); K. Sakoda, Phys. Rev. B 55, 15345 (1997).

[10] K. Kurokawa, IRE Trans. Microwave Theory Tech. 6, 178 (1958).
[11] K. Kurokawa, An Introduction to the Theory of Microwave Circuits (Academic Press, New York, 1969).

[12] W. K. H. Panofsky and M. Phillips, Classical Electricity and Magnetism (Addison-Wesley, Massachusetts, 1962), 2nd ed., p. 218.

[13] T. Weiland, M. Bartsch, U. Becker, M. Bihn, U. Blell, M. Clemens, M. Dehler, M. Dohlus, M. Drevlak, X. Du, R. Ehmann, A. Eufinger, S. Gutschling, P. Hahne, R. Klatt, B. Krietenstein, A. Langstrof, P. Pinder, O. Podebrad, T. Pröpper, U. van Rienen, D. Schmidt, R. Schuhmann, A. Schulz, S. Schupp, P. Schütt, P. Thoma, M. Timm, B. Wagner, R. Weber, S. Wipf, H. Wolter, and Z. Min, in Computational Accelerator Physics, edited by Joseph J. Bisognano and Alfred A. Mondelli, AIP Conf. Proc. No. 391 (American Institute of Physics, New York, 1997), pp. 65-70.

[14] J. Urakawa, in Proceedings of the Seventh European Particle Accelerator Conference, Vienna, 2000 (Austrian Academy of Sciences Press, Vienna, Austria, 2000), pp. 63-67.

[15] S. Sakanaka, F. Hinode, K. Kubo, T. Higo, J. Urakawa, and T. Rizawa, in Proceedings of the International Linac Conference, Tsukuba, Japan, 1994 (KEK, Tsukuba, Japan, 1994), pp. 281-283. 